\title{
Prevalence of Polycystic Ovary Syndrome and its Associated Risk Factors among Adolescent and Young Girls in Ahmedabad Region
}

\author{
N. A. Desai ${ }^{1}$, R. Y. Tiwari², S. S. Patel ${ }^{2, *}$ \\ ${ }^{1}$ Cliantha Research Centre, Ahmedabad, Gujarat, INDIA. \\ ${ }^{2}$ Department of Pharmacology, Institute of Pharmacy, Nirma University, Ahmedabad, Gujarat, INDIA.
}

\begin{abstract}
Objective: The aim of the present study was to determine prevalence, etiology of PCOS and its association with various factors in school going girls in Ahmadabad region. Study design: A cross-sectional communitybased study was conducted among the school going girls in five schools of Ahmedabad Gujarat region selected by cluster sampling. A total of 881 school going girls of age 13-18 years were involved in study. PCOS was diagnosed based on Rotterdam criteria. The association of PCOS with various factors were assessed. Estimation of Insulin, testosterone and LH was carried out. SF12 questionnaire was used for determination of quality of life. Data analysis was performed using the SPSS 9.0 PC package. Result: Out of 881 girls, 119 (13.54\%) girls were found to have PCOS, as defined by the Rotterdam criteria. The higher \% of PCOS girls belong to upper socioeconomic class (45.37). The highest \% of PCOS girls were found to have family history of diabetes mellitus (37.81). The significant correlation was found between life style factors and PCOS girls $(p \leq 0.05)$. The levels of $\mathrm{LH}$ and testosterone were found to be statistically significant in PCOS girls. There is higher percentage of girls are having poor quality of life as compared to normal girls. Conclusion: Obesity, socioeconomic status, family history, insulin resistance are the risk factors associated with PCOS. Hirsutism, acne, dysmenorrhea and oligomenorrhea were most common phenotype observed in PCOS.
\end{abstract}

Key words: Body Mass Index, Hirsutism, Hyperandrogenesim, Polycystic Ovarian Syndrome (PCOS), Rottedram criteria.

\section{INTRODUCTION}

Gynecological diseases are very common and it develops mostly at adolescence age by modification in physical nature, psychological factors and lifestyle. It gets worsen sometimes and develop sub fertility or infertility in women because of avoidance and unaware behavior of women and girls toward symptoms. One of them is polycystic ovarian syndrome (PCOS) which is commonly known as Stein Leventhal Syndrome is a gynecological endocrine disorder that occurs at any reproductive age by alteration in level of hormones. It is affecting approximately $5 \%-10 \%$ of all females and $4-6 \%$ of adolescent girls and young women worldwide. The abnormal level of hormones can make the ovaries to produce more eggs which, leads to disruption of the regular process of ovulation and starts formation of numerous cysts in ovaries and the ovaries become large and studded on ultrasound examination. It begins as early as in teenagers and mostly affect adult girls at childbearing age. ${ }^{1}$

The characteristics of PCOS includes increased secretion of androgen level (hyperandrogenesim) and gonadotropin releasing hormone $(\mathrm{GnRH})$ that leads to menstrual irregularity, hirsutism, and infertility. PCOS women may produce slightly large amount of male sex hormone, androgen than normal women. It can be diagnosed at all the phases of life that girls having 8-9 years of age through postmenopausal females. Amenorrhea is the
DOI: 10.5530/ijopp.11.3.27

Address for correspondence: S. S. Patel,

Department of Pharmacology, Institute of Pharmacy, Nirma University, Ahmedabad- 382481, Gujarat, INDIA. Phone no: 9099937466 Email Id: snehal.patel@nirmauni.ac.in

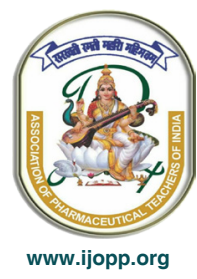


most common problem of PCOS in young girls which was first described in 1935 by Stein and Leventhal.

A study finding showed that adolescent girls with PCOS have an increased risk of the metabolic syndrome associated with increase in androgen levels independent of obesity and insulin resistance. ${ }^{2}$ PCOS has metabolic characteristics that involve prominent defects in release of insulin and $\beta$-cell function that lead to a substantially increased risk of glucose intolerance and diabetes mellitus. Obesity is also common feature in women with PCOS. Family history of obesity, diabetes mellitus, thyroid diseases, PCOS strongly supports a genetic susceptibility to this disorder. At present lifestyle, food habits, environmental exposure to toxins and stress have also contributed to the development of PCOS. Originally, PCOS is diagnosed in women at child-bearing age due to the presence of infertility but nowadays health care professionals are realizing that the teenager girls are also presenting with PCOS and the associated health concerns are menstrual irregularities, obesity, type 2 diabetes, cardiovascular disease and evidence of hyperandrogenesim (hirsutism and acne) with increasing prevalence. The severity of symptoms varies from girl to girl and women to women. ${ }^{3}$ At presently PCOS is not curable but treatment is available to alleviate the symptoms by either cyclic control of irregular menstruation cycles or by the avoidance of the long-term complications associated with obesity, insulin resistance, glucose intolerance, and type 2 diabetes. This is the first both urban and rural community based study in city of Ahmedabad, Gujarat, estimating the prevalence of PCOS among relatively younger population with a much lower mean age (13-18 years) unlike reported earlier. This is the first community based study conducted in schools among relatively younger population in the largest city of Gujarat, Ahmedabad for estimating the prevalence and early diagnosis of PCOS with association of various risk factors and also to evaluate how PCOS impact on quality of life on girls because community based data is not widely available of PCOS in India for younger population of girls and maximum prevalence studies in India are done in hospital set-ups rather than in school going girls.

\section{MATERIALS AND METHODS}

This is cross sectional, multicentric, prospective study to determine the prevalence and early diagnosis of PCOS in 963 adolescent girls which are selected from 5 urban and rural schools of Ahmedabad, the largest city of Gujarat. The duration for the collection of data was from September 2014 to April 2015. The Eligibility criteria were: school going girls of age 13- 18 years both inclusive, subjects with no history of hypolactenemia, congenital adrenal hyperplasia, thyroid disease, or other causes of amenorrhea, Cushing syndrome, androgen secreting neoplasm, tuberculosis, endometritis, were selected for the study. Out of 963 girls, study was done on 900 girls amongst 881 were given correct answers. Diagnosis of disease was done on the basis of Rotterdam Criteria. The study proposal was approved by Institutional Ethics Committee. The sample size was calculated based on the prevalence of PCOS in India and population of adolescent girls in Ahmedabad. The information was gathered on socio demographic data, menarcheal age, menstrual cycle pattern, premenstrual symptoms, and associated menstrual complications and family medical history (e.g. Diabetes, thyroid, polycystic ovarian syndrome). Weight and height were measured by standard protocol and calibrated instruments and Body mass index (BMI) was calculated. Hirsutism was assessed by Ferriman-Gallwey method based on hair growth in nine regions (i.e. upper lip, chin, chest, upper and lower abdomen, thighs, upper and lower back, and upper arms). Severity of acne was done observationally by Global acne scoring system. Obesity was assessed by to collect information about menarche age, type of diet consumed by girls. SF 12 validated questionnaire was used for taking information about health related quality of life which was also filled by girls or their parents. Blood tests were done for the biochemical estimation of hormones like insulin resistance, testosterone and Luteinizing hormone (LH) for determining of hormonal imbalances.

\section{Statistical Analysis}

Analysis was done after the collection of data, by using various statistical parameters such as Mean, Standard Deviation, percentage, correlation coefficient and chi square test method. All hypothesis tests were considered statistically significant two sided, $P \leq 0.05$ and $P \leq 0.10$. Data analysis was performed using the SPSS 9.0 PC package.

\section{RESULTS}

A total number of 881 girls were screened, 119 (13.54\%) were found to have PCOS as defined by the Rotterdam criteria. The mean age of PCOS girls was $15.73 \pm 1.28$ and the highest prevalence of PCOS was found at age group of 17 (30.25\%) (Table 1).

The mean age of first menarche was found to be 12.36 \pm 2.75 in PCOS girls and the prevalence of menstrual irregularity was found higher in PCOS girls (29.41\%) as compared to non PCOS girls (12.59\%). This shows 
Table 1: Age wise distribution of school going girls in PCOS and non PCOS girls.

\begin{tabular}{ccccc} 
& \multicolumn{3}{c}{ NO PCOS } & \multicolumn{2}{c}{ PCOS } \\
\cline { 2 - 5 } Age & $\begin{array}{c}\text { Total number } \\
(\mathbf{N}=\mathbf{7 6 2})\end{array}$ & $\begin{array}{c}\text { Percentage } \\
(\%)\end{array}$ & $\begin{array}{c}\text { Total number } \\
\mathbf{( N = 1 1 9 )}\end{array}$ & $\begin{array}{c}\text { Percentage } \\
(\%)\end{array}$ \\
\hline 13 & 55 & 6.24 & 7 & 5.88 \\
14 & 195 & 22.13 & 17 & 14.28 \\
15 & 225 & 25.53 & 20 & 16.80 \\
16 & 165 & 18.72 & 34 & 28.57 \\
17 & 96 & 10.89 & 36 & 30.25 \\
18 & 26 & 2.95 & 5 & 4.20 \\
\hline
\end{tabular}

Table 2: Age wise prevalence of menstrual complications in PCOS girls.

\begin{tabular}{|c|c|c|c|c|c|c|}
\hline Menstrual Complications & $\begin{array}{c}\text { Age } 13 \\
(\%)\end{array}$ & $\begin{array}{c}\text { Age } 14 \\
(\%)\end{array}$ & $\begin{array}{c}\text { Age } 15 \\
(\%)\end{array}$ & $\begin{array}{c}\text { Age } 16 \\
(\%)\end{array}$ & $\begin{array}{c}\text { Age } 17 \\
(\%)\end{array}$ & $\begin{array}{c}\text { Age } 18 \\
(\%)\end{array}$ \\
\hline Dysmenorrhea & 28.57 & 41.17 & 90 & 64.7 & 69.44 & 100 \\
\hline Amenorrhea & 14.28 & 0 & 0 & 8.82 & 2.77 & 0 \\
\hline Menorrhagia & 28.57 & 35.29 & 30 & 29.41 & 13.88 & 20 \\
\hline Metrorrhagia & 0 & 23.52 & 35 & 20.58 & 13.88 & 0 \\
\hline Hypomenorrhea & 0 & 0 & 25 & 8.82 & 13.88 & 0 \\
\hline
\end{tabular}

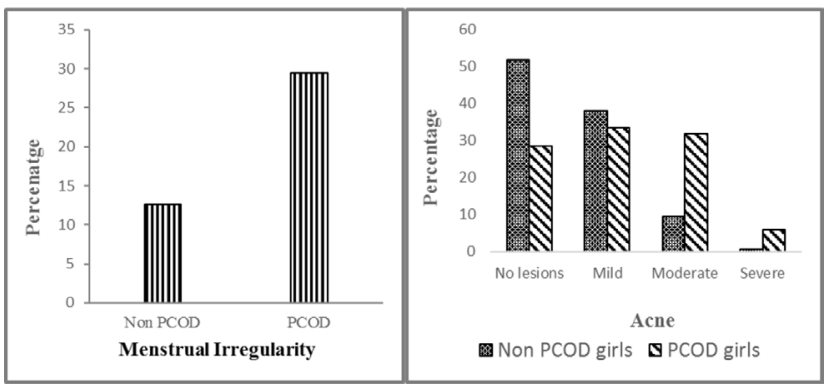

Figure 1: Prevalence of menstrual irregularity and Acne in non PCOS and PCOS.

significant correlation between menstrual irregularity and PCOS. The prevalence of PCOS girls was having higher severe hirsutism (47.05\%) and over hirsutism (25.21\%) than non-PCOS girls (Figure 1). Thus there was found significant correlation of hirsutism with PCOS.

The results showed that PCOS girls were having higher prevalence of moderate $(31.93 \%)$ and sever $(5.88 \%)$ acne than non-PCOS girls, $9.44 \%, 0.65 \%$ respectively. Severe acne at age group of $14(11.76 \%)$ in PCOS girls. The higher prevalence of mild and moderate acne were having at age group of $17(41.66 \%)$ and $18(60 \%)$ respectively in PCOS girls. So, the occurrence of acne is significant $(P \leq 0.05)$ in PCOS girls when compared to non PCOS girls. The highest prevalence of PCOS girls were having dysmenorrheal at age 18. The higher prevalence of amenorrhea, menorrhagia, metrorrhagia and hypomenorrhea were having at age 13, age 14, age 15 , age 25 respectively. Thus, the occurrence of dysmenorrheal and amenorrhea in PCOS girls are not significant $(P \leq 0.05)$ when compared with normal population (Table 2$)$.

The higher prevalence of overweight in PCOS girls was found to be at age $18(60 \%)$ and obese was found to be at age $16(20.58 \%)$. The highest percentage of PCOS girls were having family history of diabetes mellitus (37.81\%), thyroid (20.16\%) and hypertension (17.64\%) as compared to normal girls having $4.06 \%, 6.43 \%, 18.76 \%$ respectively. So, family history of diabetes mellitus and thyroid were statically differ between PCOS and non- PCOS girls but (Figure 2)

Family history of hypertension was not statistically much more different in PCOS and non PCOS girls. Based on body mass index, blood pressure was found slight correlation with PCOS but not significantly (Figure 3).

Based on the socioeconomic status, it was found that higher percentage of PCOS girls belong to upper socioeconomic class $(45.37 \%)$ as compared to non PCOS girls $(35.43 \%)$. Hence, upper class girls are more prevalent to PCOS than other class (Figure 4).

The mean of LH and testosterone was not found statistically significant (Table 3). 


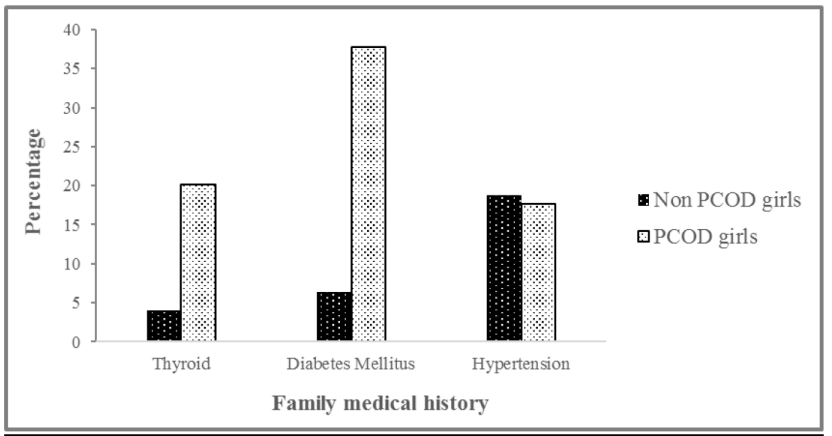

Figure 2: Comparison of family medical history in non PCOS and PCOS girls.

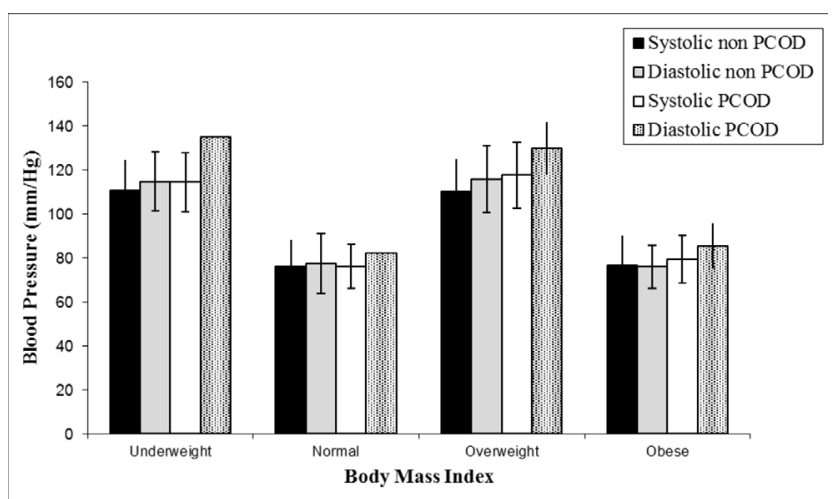

Figure 3: Comparison of blood pressure among PCOS and Non-PCOS girls.

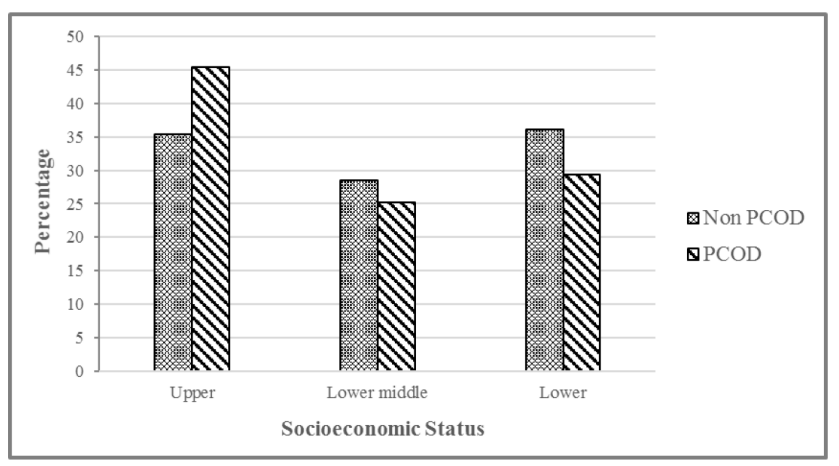

Figure 4: Association of PCOS with socioeconomic status in girls.

\begin{tabular}{|c|c|c|}
\hline Biochemical estimation & $\begin{array}{c}\text { Non PCOS } \\
(\mathrm{N}=10) \\
\text { Mean } \pm \text { SD }\end{array}$ & $\begin{array}{c}\text { PCOS } \\
(\mathrm{N}=16) \\
\text { Mean } \pm \text { SD }\end{array}$ \\
\hline $\mathrm{LH}$ & $2.34 \pm 0.81$ & $2.15 \pm 1.32$ \\
\hline Testosterone & $21.07 \pm 5.46$ & $23.38 \pm 6.54$ \\
\hline
\end{tabular}

The higher percentage of PCOS girls were not done physical exercise and not participating in sports as compared to non PCOS girls and that may be lead to obesity and further causes in PCOS girls (Table 4).
Table 4: Association of PCOS with life style factors in girls diagnosed with PCOS.

\begin{tabular}{|c|c|c|}
\hline Daily activities & $\begin{array}{c}\text { Non PCOS } \\
(\%)\end{array}$ & $\begin{array}{l}\text { PCOS } \\
(\%)\end{array}$ \\
\hline \multicolumn{3}{|c|}{ Participates in sports } \\
\hline Yes & 67.97 & 38.65 \\
\hline No & 32.02 & $61.34^{*}$ \\
\hline \multicolumn{3}{|c|}{ Physical exercise } \\
\hline Yes & 29.92 & 33.61 \\
\hline No & 70.07 & 66.38 \\
\hline \multicolumn{3}{|c|}{ Sleeping habits in afternoon } \\
\hline Yes & 40.68 & 59.66 \\
\hline No & 59.31 & 40.33 \\
\hline \multicolumn{3}{|c|}{ Diet Habit } \\
\hline Vegetarian & 91.07 & 94.95 \\
\hline Non vegetarian & 6.03 & 4.2 \\
\hline Eggeterian & 2.88 & 0.84 \\
\hline \multicolumn{3}{|c|}{ Taking junk food } \\
\hline Everyday & 6.29 & 10.92 \\
\hline Once in week & 29.52 & 30.25 \\
\hline Twice in month & 27.82 & 23.52 \\
\hline Once in month & 26.64 & 27.73 \\
\hline Not at all & 9.71 & 7.56 \\
\hline
\end{tabular}

SF12 health survey showed that higher percentage of girls are having problems related to general health and doing moderate activities like moving table, pushing vaccum cleaner, bowling, or playing golf, climbing several flights of stairs, feeling downhearted and blue, calm and peaceful, does not have much energy as compared to normal girls (Table 5).

\section{DISCUSSION}

In 2003, the Rotterdam European Society for Human Reproduction/American Society of Reproductive Medicine (ESHRE/ASRM) proposed three diagnosis criteria include oligo and/or anovulation, clinical and/or biochemical hyperandrogenesim and polycystic ovaries on ultrasonography. The most recent set of criteria was defined by Androgen Excess Society (AES) in 2006, which has recommended the following diagnostic criteria for PCOS: hirsutism and/or hyperandrogenism, oligoand anovulation and/or polycystic ovaries and other androgen excess or related disorders should be excluded. ${ }^{3}$

PCOS is a chronic condition with obesity, hirsutism, acne, menstrual irregularities and transition over time into infertility and metabolic complications. ${ }^{1}$ This is the first cross sectional study carried out in Ahmedabad city of Gujarat to find prevalence of PCOS among relatively 


\section{Table 5: Comparison of Health Related Quality of Life in girls diagnosed with PCOS.}

\begin{tabular}{|c|c|c|}
\hline Health Related Quality of Life & $\begin{array}{l}\text { Non PCOS } \\
(\%)\end{array}$ & $\begin{array}{l}\text { PCOS } \\
(\%)\end{array}$ \\
\hline \multicolumn{3}{|l|}{ General Health } \\
\hline Excellent & 16.53 & 12.6 \\
\hline Very good & 24.67 & 27.73 \\
\hline Good & 25.32 & 21 \\
\hline Fair & 31.49 & 34.45 \\
\hline Poor & 1.96 & $4.2^{*}$ \\
\hline \multicolumn{3}{|c|}{$\begin{array}{c}\text { Moderate activities like moving table, pushing vacuum cleaner, } \\
\text { bowling, or playing golf }\end{array}$} \\
\hline Yes, Limited a lot & 21.65 & 40.33 \\
\hline Yes, Limited a little & 33.2 & 33.61 \\
\hline No, Not limited at all & 45.14 & 26.05 \\
\hline \multicolumn{3}{|l|}{ Climbing several flights } \\
\hline Yes, Limited a lot & 21.39 & 36.13 \\
\hline Yes, Limited a little & 36.22 & 38.65 \\
\hline No, Not limited at all & 42.38 & 25.21 \\
\hline \multicolumn{3}{|l|}{ Any problem during work done } \\
\hline Yes & 42.91 & 34.45 \\
\hline No & 57.08 & 65.54 \\
\hline \multicolumn{3}{|l|}{ Emotional problems } \\
\hline Yes & 36.61 & 34.45 \\
\hline No & 63.38 & 65.54 \\
\hline \multicolumn{3}{|l|}{ Did not do work or other activities carefully } \\
\hline Yes & 35 & 63.02 \\
\hline No & 59.18 & 36.97 \\
\hline \multicolumn{3}{|l|}{ Pain interfere during work } \\
\hline Not at all & 33.07 & 44.53 \\
\hline A little bit & 25.98 & 28.57 \\
\hline Moderately & 29 & 18.48 \\
\hline Quite a bit & 10.89 & 5.88 \\
\hline Extremely & 1.04 & 2.52 \\
\hline \multicolumn{3}{|l|}{ Feel calm and peaceful } \\
\hline All of the time & 8.79 & 6.72 \\
\hline Most of the time & 25.45 & 31.09 \\
\hline A good bit of the time & 10.36 & 10.92 \\
\hline Some of the time & 17.58 & 13.44 \\
\hline A little of the time & 25.32 & 22.68 \\
\hline None of the time & 12.46 & 15.12 \\
\hline \multicolumn{3}{|l|}{ Feel energetic } \\
\hline All of the time & 16.01 & 9.24 \\
\hline Most of the time & 25.59 & 25.21 \\
\hline A good bit of the time & 12.33 & 10.08 \\
\hline Some of the time & 17.19 & 10.92 \\
\hline A little of the time & 16.79 & 21.00 \\
\hline None of the time & 12.07 & 23.52 \\
\hline Feel downhearted and blue & 11.67 & 23.52 \\
\hline All of the time & 28.34 & 12.60 \\
\hline Most of the time & 10.36 & 10.08 \\
\hline A good bit of the time & 16.92 & 10.92 \\
\hline Some of the time & 16.4 & 21.00 \\
\hline A little of the time & 16.27 & 21.84 \\
\hline None of the time & 11.67 & 23.52 \\
\hline
\end{tabular}


younger population (13-18 years) based on the Rotterdam criteria. The prevalence of PCOS in sample of adolescent girls in Ahmedabad was found to be $13.54 \%$. However, the prevalence of PCOS in another study in Rasht population was found to be $11.34 \%$ which was almost similar to the results of our study ${ }^{2}$ but slightly higher as compared to other previously reported studies.,

According to the history and physical examination, PCOS was diagnosed in adolescents who presented with both menstrual dysfunction and clinical hyperandrogenism (hirsutism, acne). The higher rate of PCOS in our study was attributed to the fact that we used Rotterdam criteria instead of NIH. In this, study it was found that higher percentage of girls with PCOS were having menstrual irregularity as compared to normal girls which was similar to other reported investigation. ${ }^{7}$ In our study, the prevalence of hirsutism in PCOS girls was found 25.21\% but it was variant with different population. ${ }^{6,5}$ Thus, prevalence of hirsutism in PCOS varies by ethnicity. One third of women with PCOS, particularly younger women, demonstrate acne ${ }^{7}$ by excess of androgens hormone level. The prevalence of acne in this study was found to be $5.88 \%$ in PCOS girls which was slightly lower than previous studies such as in Spain population, it was found to be $7.1 \%{ }^{8}$

In PCOS patient, level of male sex hormone, testosterone is slightly increase by increase Luteinizing hormone which can suppress normal menstruation and ovulation. ${ }^{9}$ In this study there was a slight increase in testosterone and $\mathrm{LH}$ levels in PCOS girls but it could not be able to confirm PCOS as per Rotterdam criteria.

Menstrual disorders were a common problem in adolescents which were the source of anxiety for the patients and the families. In our study, the prevalence rate of dysmenorrhea, oligomenorrhea, menorrhagia, metrorrhagia in PCOS girls was statistically different from normal group coincide with other reported studies. ${ }^{5,10}$ During the past decades, the increasing prevalence of PCOS in children and adolescents has coincided with the rise in obesity, similar to adults. ${ }^{11}$ The prevalence of overweight and obesity in PCOS girls were higher than non-PCOS girls which was similar with other study report. ${ }^{12}$ Thus, there was significant correlation between obesity and PCOS.

Family history of diabetes mellitus and hypothyroidisms were having higher association with PCOS. Similar results were obtained in this study. But the family history of hypertension was not significantly different with normal population which was also accompanied with other reported studies. ${ }^{13}$ Previous studies had reported that the probability of finding a metabolic disorder in the families of PCOS patients is 2.7 fold higher than in the control group families. ${ }^{14}$ Thus, PCOS is more prevalent in girls whose parents and grandparents were having metabolic disorders than in those of normal parents. Earlier studies have reported that the prevalence of hypertension is three times higher in women with PCOS as compared to normal. ${ }^{15}$ In our study results showed that obese girls having PCOS were having higher blood pressure as compared to non-obese PCOS girls which was almost similar to the other reported studies. ${ }^{15}$ Thus, Higher Blood pressure was directly correlated with obesity and PCOS. Therefore, Adolescents obese girls with PCOS were at higher risk of hypertension and possible mechanism was either higher rennin plasma activity ${ }^{16}$ or upregulation of endothelin-1.

The association of PCOS with socioeconomic status (SES) might shed light on the role of the environment in the development of PCOS. In our study we observed that Upper SES has significant association with PCOS as similar with previous research studies had shown that individuals with upper SES were more risk for developing of obesity and insulin resistance which was highly correlated with the pathogenesis of PCOS. ${ }^{17}$

In this study we also focused on girl's daily activities, eating habits and unhealthy behaviors. There was no significant difference showed in terms of diet and unhealthy behaviors but in terms of physical activities showed significant relation with PCOS which was higher in PCOS as compared to normal population. Few studies have been conducted on lifestyle and nutritional habits of people with PCOS and showed appropriate lifestyle modifications and increasing physical activity considered as the first line treatment for PCOS patients. ${ }^{18}$

In this study it was evaluated that girls with PCOS having significantly lower SF-12 score as compared to non-PCOS girls which was correlate with study carried out on Australian PCOS population. ${ }^{26}$ Thus, PCOS has a negative impact upon health related quality of life (HRQoL). Obesity and Blood pressure appeared to exert greatest negative influence upon HRQoL in PCOS. Thus, Special attention should be paid to overweight and obese girls as they were having a highest possibility of PCOS.

\section{CONCLUSION}

In conclusion, the present study suggests that PCOS is an emerging disorder associated with many health consequences like hirsutism, acne, menstrual irregularities which were common endocrine disorders show 
in adolescent girls. Family history of diabetes and hypothyroidism were important associated risk factors with PCOS. Awareness of earlier diagnosis of PCOS and interventions must be increased among health care providers and adolescent girls because it could provide opportunity to treat this disease and prevent future morbidities. Special attention should be paid to overweight and obese girls as they were having highest possibility of PCOS but it could treat by lifestyle modification. Thus early detection of the syndrome based on clinical findings (mainly oligo- or amenorrhea) offers an opportunity for early intervention to prevent or limit the impact of cutaneous and reproductive symptoms, and the long-term effects of metabolic disturbances (Franks, 2008).

\section{ACKNOWLEDGEMENT}

The authors would like to acknowledge the funding provided by Institute of Pharmacy, Nirma University in the form of post-graduate contingency.

\section{CONFLICT OF INTEREST}

The authors declare no conflict of interest.

\section{ABBREVIATIONS}

PCOS: Polycystic Ovarian Syndrome; GnRH: gonadotropin releasing hormone; BMI: Body mass index; LH: Luteinizing hormone; ESHRE: European Society for Human Reproduction; ASRM: American Society of Reproductive Medicine; AES: Androgen Excess Society; SES: Socioeconomic status; HRQoL: Health related quality of life.

\section{SUMMARY}

This study signifies that PCOS is an emerging disorder during adolescence and its early detection will provide opportunity to target the group for promoting healthy and better lifestyles. So special awareness should be taken to limit the impact of cutaneous and reproductive symptoms and the long-term effects of metabolic disturbances.

\section{REFERENCES}

1. Franks S. Polycystic ovary syndrome in adolescents. International Journal of Obesity. 2008;32(7):1035-41.

2. Nidhi R, Padmalatha V, Nagarathna R, Amritanshu R. Prevalence of Polycystic Ovarian Syndrome in Indian Adolescents. Journal of Pediatric and Adolescent Gynecology. 2011;24(4):223-7.

3. Arora B, Arora V, Patel S. Polycystic Ovarian Syndrome:Anawareness guide for women. Journal of Pharmacy Research. 2014;8(7):884-92.

4. Asgharnia M, Mirblook F, Soltani MA. The Prevalence of Polycystic Ovary Syndrome (PCOS) in High School Students in Rasht in 2009 According to NIH Criteria.

5. Diamanti-Kandarakis E, Kouli CR, Bergiele AT, Filandra FA, Tsianateli TC, Spina GG, et al. A Survey of the Polycystic Ovary Syndrome in the Greek Island of Lesbos: Hormonal and Metabolic Profile. The Journal of Clinical Endocrinology and Metabolism. 1999;84(11):4006-11.

6. International Journal of Fertility and Sterility. 2011;4(4):156-9.

7. Asunción M, Calvo RM, San MJL, Sancho J, Avila S, Escobar-Morreale HF. A prospective study of the prevalence of the polycystic ovary syndrome in unselected Caucasian women from Spain. J Clin Endocrinol Metab. 2000;85(7):2434-8.

8. Van HMH, Voorhorst FJ, Kaptein MB, Hirasing RA, Koppenaal C, Schoemaker $\mathrm{J}$. Endocrine features of polycystic ovary syndrome in a random population sample of 14-16 year old adolescents. Hum Reprod. 1999;14(9):2223-9.

9. Azziz R, Carmina E, Dewailly D, Diamanti-Kandarakis E, Escobar-Morreale HF, Futterweit W, et al. Positions statement: criteria for defining polycystic ovary syndrome as a predominantly hyperandrogenic syndrome: an Androgen Excess Society guideline. J Clin Endocrinol Metab. 2006;91(11):4237-45.

10. Ehrmann DA. Polycystic Ovary Syndrome. New England Journal of Medicine. 2005;352(12):1223-36.

11. Hedley AA, Ogden CL, Johnson CL, Carroll MD, Curtin LR, Flegal KM. Prevalence of overweight and obesity among US children, adolescents, and adults, 1999-2002. JAMA. 2004;291(23):2847-50.

12. Ben SHL, Ben SHS, Bouzid C, Younsi N, Smida H, Bouguerra R, et al. [Hypertension in polycysticovary syndrome].Arch Mal CoeurVaiss. 2006;99(7-8): 687-90.

13. Benítez R, Sir-Petermann T, Palomino A, Angel B, Maliqueo M, Pérez F, et al. [Prevalence of metabolic disorders among family members of patients with polycystic ovary syndrome]. Rev Med Chil. 2001;129(7):707-12.

14. Sinha U, Sinharay K, Saha S, Longkumer TA, Baul SN, Pal SK. Thyroid disorders in polycystic ovarian syndrome subjects: A tertiary hospital based cross-sectional study from Eastern India. Indian J Endocrinol Metab. 2013;17(2):304-9.

15. Rahmanpour H, Jamal L, Mousavinasab SN, Esmailzadeh A, Azarkhish K. Association between polycystic ovarian syndrome, overweight, and metabolic syndrome in adolescents. J Pediatr Adolesc Gynecol. 2012;25(3):208-12.

16. Reaven GM, Lithell H, Landsberg L. Hypertension and associated metabolic abnormalities--the role of insulin resistance and the sympathoadrenal system. N Engl J Med. 1996;334(6):374-81.

17. Uncu G, Sözer MC, Develioğlu O, Cengiz C. The role of plasma renin activity in distinguishing patients with polycystic ovary syndrome (PCOS) from oligomenorrheic patients without PCOS. Gynecol Endocrinol. 2002;16(6):447-52.

18. Merkin SS, Azziz R, Seeman T, Margalit RC, Daviglus M, Kiefe C, et al. Socioeconomic Status and Polycystic Ovary Syndrome. J Women's Health. 2011;20(3):413-9.

19. Ching HL, Burke V, Stuckey BGA. Quality of life and psychological morbidity in women with polycystic ovary syndrome: body mass index, age and the provision of patient information are significant modifiers. Clin Endocrinol (Oxf). 2007;66(3):373-9.

20. Richardson MR. Current perspectives in polycystic ovary syndrome. Am Fam Physician. 2003;68(4):697-704. 